QUIPURAMAYOC $\mid$ Revista de la Facultad de Ciencias Contables

Vol. 20 N.o 37 pp. 40-53 (2012) UNMSM, Lima, Perú

ISSN: 1560-9103 (versión impresa) / ISSN: 1609-8196 (versión electrónica)

\title{
LA CIENTIFICIDAD EN LA DISCIPLINA CONTABLE
}

THE SCIENTIFICITY IN THE ACCOUNTING DISCIPLINE

Arturo Torres Gallardo*

[Recepción: Julio de 2012/ Conformidad: Agosto de 2012]

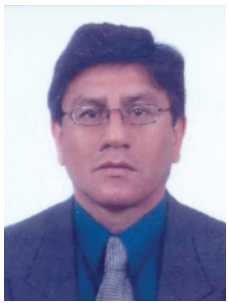

\section{RESUMEN}

En la actualidad, la consideración de un conocimiento como científico o no, resulta de aplicar criterios de carácter convencional establecidos por los hombres de forma subjetiva y en ocasiones arbitraria. Como menciona Montes (2006), los cánones de la ciencia tienen vigencia espacio-temporal, los criterios que en una época o lugar pueden calificarse como científicos, en otro tiempo o lugar pueden ser metafísicos. El objetivo de este artículo es dar a conocer los elementos o componentes de una ciencia, los cuales han sido deducidos de la literatura revisada, observando cuidadosamente las diferentes definiciones de ciencia que cada corriente de pensamiento expresa. Ello evidencia el proceso evolutivo de la ciencia, como consecuencia de las distintas corrientes de pensamiento existentes, las cuales conforme a su perspectiva, podían dar a conocer las diferentes formas de comprender la naturaleza, los fenómenos, la realidad, etc., precisamente por el uso del método para obtener el conocimiento científico. Las fuentes bibliográficas consultadas, están relacionadas con la Filosofía de la Ciencia. Los métodos utilizados están constituidos básicamente por: histórico, analítico-sintético, deductivo, hermenéutico. Se encontró que independiente de cual fuere la corriente de pensamiento que privilegia tal o cual método, todas ellas han mostrado en su definición de ciencia elementos o componentes comunes, a lo largo de su proceso evolutivo y que podríamos decir que caracterizan a toda ciencia. Hemos

\begin{abstract}
At present, the consideration of knowledge as scientific or not, is the result of applying conventional character criteria established by men in a subjective and sometimes arbitrary way. As mentioned by Montes (2006), the canons of science are effective space-time, the criteria at a time or place can be described as scientific, in another time or place can be metaphysical. The aim of this scientific article is to know the elements or components of a science, which have been deducted from the literature reviewed, carefully observing the different definitions of science that every school of thought says. This shows the evolutionary process of science, as a result of the different schools of thought exist, which according to their view, could raise awareness of different ways of understanding the nature, phenomena, reality, etc., precisely because using the method of obtaining scientific knowledge. The bibliographical sources consulted, are related to the Philosophy of Science. The methods are basically based on: historical, analytic-synthetic, deductive, hermeneutic. We found that regardless of the independent school of thought that favors this or that method, all have shown in their definition of science elements or common components along their evolutionary process and we might say it characterizes all science. We have identified three components of all knowledge: the object of study, the method and predictability.
\end{abstract}

* Doctorante en Ciencias Económicas y Sociales en la Universidad Johannes Kepler, Linz-Austria; Contabilidad y Finanzas de la Escuela de Post Grado de la Universidad Nacional de Trujillo, Perú. E-mail:atorresg42@hotmail.com 
identificado tres elementos componentes de toda ciencia: el objeto de estudio, el método y la capacidad de predicción.

Palabras claves: Filosofía, ciencia, paradigma, método, capacidad de predicción.

\section{INTRODUCCIÓN}

En sus orígenes la ciencia y la filosofía constituían una sola cosa. Sólo la Filosofía abarcaba todo el saber y todo el contenido de lo que hoy llamamos ciencia. La ciencia se dividía en el saber sobre el ser en cuanto tal y en géneros particulares del ser. Pero lo que movía al hombre a estudiar era el deseo de saber, de saber cómo son las cosas.

Considerar a la ciencia como un saber no filosófico es un resultado de mucho tiempo, la ciencia moderna que hoy conocemos surgió entre los S. XVI y S. XVII separándose de la filosofía; y un rasgo del pensamiento moderno fue la intención de aproximar la filosofía y la ciencia. Filosofía y ciencia no solo no se oponen, sino que se encuentran como dos extremos, como en dos polos entre los que se desarrolla todo el pensamiento racional de la humanidad.

A través del desarrollo histórico se han desarrollado dos grandes tradiciones respecto a lo que debe entenderse por ciencia: a) aquella que la entiende como la explicación que la razón otorga a los diversos fenómenos investigados, b) aquella que la entiende como la explicación causal de los hechos. El concepto semántico de ciencia que se tenía antiguamente es indudablemente distinto al que se tiene en la actualidad. Por ello, muchas disciplinas hoy se encuentran actualmente en proceso de ser consideradas ciencia.

El problema que evidencia la disciplina contable es similar a la confusión que existía entre filosofía y ciencia. La contabilidad suele ser confundida frecuentemente con la téc-
Keywords: Philosophy, science, paradigm, method, predictability

nica de la teneduría de libros. Esta técnica, comprende básicamente el registro, procesamiento y resumen de los datos con la finalidad de obtener información contable. De igual modo, la contabilidad también ha sido considerada por algunos como tecnología.

Esta situación nos ha motivado para que a partir de la literatura y de las lecturas revisadas, se pretenda responder el estado de cientificidad de la disciplina contable. En tal sentido, es el objetivo del presente artículo, explicar el nivel de cientificidad alcanzado por la disciplina contable, a través de la identificación dentro de la ciencia de los elementos teóricos componentes, formulándonos para ello la hipótesis: La contabilidad es ciencia debido a que posee los elementos teóricos básicos de toda ciencia: objeto de estudio, método y capacidad de predicción. Por tanto, el presente estudio se justifica por la necesidad de contribuir teóricamente a establecer criterios para identificar elementos o condiciones básicas que una disciplina debe poseer para considerarse una ciencia y tendrá un interés práctico porque permitirá culminar con la vieja discusión de la disciplina contable es o no ciencia.

\section{MATERIALES Y MÉTODOS}

Materiales o fuentes:

Libros y trabajos de investigación relacionados con la Filosofía de la Ciencia

Métodos

Los métodos que se utilizaron fueron: documental, histórico, deductivo, analítico-sintético, crítico o hermenéutico. 


\section{RESULTADOS}

1. La ciencia según Aristóteles: La ciencia como explicación teleológica

La definición aristotélica enumera e identifica las propiedades del conocimiento científico: Conocimiento de esencias, intenta responder siempre a ¿qué es?; conocimiento causal, se pregunta por las causas de las cosas; conocimiento necesario, en el sentido de que aquello que es, no podría no ser; conocimiento universal, entendiendo universal por aquello que es fijo, inmutable y necesario. En la explicación de las características del conocimiento científico, Fraile (s/f) no olvida la diferencia entre el orden lógico y el orden ontológico, introducido por Aristóteles para dar cabida dentro de la ciencia a las sustancias materiales del mundo sensible. Éstas aunque no necesarias ontológicamente, sí cabe hallar en ellas una necesidad lógica suficiente para elevarlas a objetos de ciencia. ¿Cómo? (sic) Mediante la actividad abstractiva de nuestro entendimiento. La existencia de estas sustancias es lo que lleva a Aristóteles a llamar la atención acerca de la diferencia de certeza que debe exigirse a cada una de las ciencias. Fraile (s/f) señala que Aristóteles conserva el concepto platónico de la ciencia como conocimiento, fijo, estable y necesario pero la necesidad la busca no en el orden ontológico ficticio y apriorístico, sino en el orden lógico. El problema de la ciencia en Aristóteles consiste en (sic) "dotar de los caracteres de fijeza, estabilidad y necesidad a los objetos particulares materiales y móviles del mundo físico, tal como son percibidos por los sentidos".

El principio del quehacer científico estaba en la observación. Se consideraba que el conocimiento es un conocimiento por causas
(Echevarria, 2002, 64) y la explicación teleológica era el objetivo final de la ciencia, lo que constituía a su vez la condición sine qua non. Lejos, pues, de tener el concepto universal como construcción apriorística, Aristóteles ofrece un producto elaborado por el entendimiento, de valor lógico.

2. La ciencia desde la perspectiva empirista: Ciencia como explicación causal

La corriente empirista se desarrolla en Gran Bretaña en parte del siglo XVII y el siglo XVIII. Es iniciada por Francis Bacon en el siglo XVI (renacimiento tardío), propone dejar de mirar al universo como un conjunto de sustancias con sus propiedades y poderes y se empieza a mirarla como un flujo de acontecimientos que suceden según leyes. Su pensamiento expresa que sólo el conocimiento sensible nos pone en contacto con la realidad. Para los empiristas, las ciencias naturales son el tipo ideal de ciencia, ya que se basa en hechos observables.

Considerar la experiencia como la única fuente válida de conocimiento. Esto indudablemente constituye otra diferencia metodológica con la visión aristotélica de ciencia. El método ideal para los empiristas es precisamente el experimental e inductivo.

Galileo Galilei (1564-1662) y el renacimiento: un nuevo concepto de ciencia

Galileo Galilei es célebre por su contribución a la ciencia y a la historia de la misma, por el descubrimiento de importantes leyes físicas: ley de la inercia, ley de la caída libre. Con ello, establece los cimientos de la mecánica moderna y un nuevo concepto de ciencia, aunque no menos importante de su aporte es el método que en ello empleó: el inductivo y el experimental. Lo esencial de su método son las fases que contiene: resolución, composición y generalización. 
Isaac Newton (1642-1727)

Su contribución a la ciencia es también muy importante ya que desarrolló la ley de la caída de Galileo, construye la teoría de la gravitación y su doctrina sobre la órbita de los planetas. Se desarrolla así un sistema cerrado de la mecánica que se considero en la edad media como única manera de ver la naturaleza, que posteriormente fuera tomada por Kant como punto de partida para su concepción del mundo físico.

Como aporte de esta corriente filosófica podemos señalar que se establece un nuevo campo de la ciencia. La ciencia del ser se resuelve en ciencia del acontecer. En opinión de Hirschberger (1968), no se pregunta ya cómo emanan los accidentes de la substancia y como debe entenderse lo que acaece partiendo de la misma sustancia; se indaga más bien la dependencia con que a la luz de la experimentación, aparecen entre sí relacionados los diversos factores de un proceso observado, sustituyéndose entonces el concepto de sustancia por el de función y el de esencia por el de ley física.

Desde la perspectiva empirista, la ciencia es pues, un conjunto de conocimientos obtenidos de una serie de hechos individuales, que han sido confirmados a través de la experimentación, sobre los cuales recae un proceso de abstracción riguroso que permite obtener conceptos generales de las cosas y las leyes de la naturaleza. Este concepto de ciencia, si bien es cierto no proporciona una certeza absoluta pero si suficiente, cuando se realiza con perfecta escrupulosidad (Marias, 1971). Adviértase aquí, que la utilización de la experiencia como medio para conocer, exige la capacidad de predicción del conocimiento científico, poniendo en evidencia un nuevo elemento en la definición de ciencia.
3. La ciencia desde la perspectiva racionalista Renato Descartes (1596-1650)

El racionalismo se caracterizará por la afirmación de que la certeza del conocimiento procede de la razón, lo que va asociado a la afirmación de la existencia de ideas innatas. Ello supondrá la desvalorización del conocimiento sensible, en el que no se podrá fundamentar el saber, quedando la razón como única fuente de conocimiento. Su mejor exponente es Renato Descartes, a quien se le conoce como el padre de la filosofía moderna y ha sido el primer y el último gran filósofo francés.

El punto de partida de la teoría gnoseológica o epistemológica de Descartes está en conseguir un sistema filosófico que haga avanzar la filosofía, que elimine toda la variedad de opiniones y teorías contrarias habidas hasta el momento. Descartes quiere lograr como buen racionalista, una filosofía que unifique sus verdades como lo hace la matemática. Según él, los errores filosóficos se deben a que no se ha utilizado un buen método para buscar la verdad.

Para Descartes el éxito de las matemáticas radica no en su estructura que hoy denominaríamos axiomática, sino en el método que utiliza. Y ese método es el método deductivo. Por tanto, queda claro que, el conocimiento de la verdad debe ir asociado a la utilización de un método. Si el conocimiento de la naturaleza es posible gracias a las matemáticas es pensable que utilizando el método que utiliza las matemáticas se pueda alcanzar la verdad y la certeza en el conocimiento de los otros aspectos de la realidad.

Empirismo y racionalismo Se podría decir que la filosofía se hace moderna con el advenimiento del empirismo, 
el cual termina con la tradición de la metafísica, es decir, ya no hay metafísica, no hay trascendencia, y sobre todo ya no hay verdades inmutables y eternas (Hirschberger, $1967,103)$. Esto constituye la fundamental diferencia que separa el empirismo del racionalismo. La valoración de la experiencia en ambas corrientes filosóficas es la principal diferencia. Para el empirismo la experiencia sensible lo es todo. Ello y sólo ello decide lo que es verdad, valor, ideal, derecho, religión. Dicha experiencia no tiene término, nunca es cosa conclusa, lo que conlleva a que no existan verdades, valores, ideas, etc. eternas, es decir, todo queda relativizado, en función del tiempo, espacio, de lo humano.

Por otro lado, para el racionalismo, la experiencia es menos consultada que en el empirismo, constituyendo solo el material, la ocasión, el motivo para conocer. Ciencia (hechos observados, experimentados) y verdad (producto de la razón) son dos cosas distintas en el entendimiento y en sus conocimientos necesarios de esencia.

\section{La ciencia en el pensamiento positivista} El positivismo es una corriente filosófica burguesa moderna que declara las ciencias concretas (empíricas) única fuente del saber verdadero y niega valor cognoscitivo a las indagaciones filosóficas (Rosental, 2005). El fundador del positivismo fue Augusto Comte (1788-1857), desde la primera mitad del siglo XIX, y fue él quien introdujo este término con que se conoce esta teoría. Históricamente, en esta corriente se distinguen tres fases en su evolución: positivismo, empiriocriticismo y neopositivismo.

La ciencia desde esta perspectiva, está compuesta por enunciados básicos o protocolares, cuya certeza viene dada por la percep- ción inmediata de los sentidos (Hashimoto, 2010). Es decir, la ciencia se define y justifica por los términos sacados de la experiencia sensible. En la forma positivista de examinar la naturaleza, las impresiones sensoriales son los datos primarios y, en consecuencia, significan realidad inmediata ${ }^{1}$. Prevalece en esta perspectiva como explicación científica la explicación causal, con la finalidad de encontrar los motivos fundamentales, la esencia matemática que permita la predicción de los fenómenos.

Esta corriente propicia el camino de diferenciación del conocimiento a través del criterio de demarcación entre lo que es o no ciencia. Los positivistas le dan un énfasis al método inductivo, que es el método de las ciencias, el cual debería servir a las demás ciencias. A esto se le llamó el monismo metodológico. En opinión de Kopnin (1966), la ciencia para los positivistas, es un sistema de proposiciones no contradictorias, que concuerdan en lo fundamental y poseen su propia concepción. Precisa que los positivistas afirman que la ciencia no investiga el mundo objetivo propiamente dicho, sino los datos de los aparatos de medición, sus anotaciones que son lo que constituyen el verdadero objeto de las ciencias exactas.

5. La ciencia desde la perspectiva del racionalismo crítico El racionalismo crítico es la base principal de la filosofía de Popper, la cual consiste en hacer una crítica a las teorías establecidas por la ciencia y se opone expresamente al positivismo lógico. El pensamiento de Popper es una oposición al empirismo basado en la naturaleza y la experiencia de los sentidos. La formación del conocimiento pasa a ser parte fundamental como un proce-

1 PLANCK, Max. En: Palacios L., Lucas; p.124. 
so evolutivo que parte de problemas y tiene intentos de solución y exclusión de intentos fallidos. Para Popper, el observacionismo de Bacon, debe referirse a la naturaleza como el comienzo y fin de las cosas, del método de deducción como la observación pura, libre de malas teorías y del error (Popper,1997, 89), la idea de depurar la mente de prejuicios es una idea ingenua y equivocada, de una mente pura pero vacía, para el racionalismo critico el conocimiento científico consiste en aprender de nuestros errores y examinarlos.

5.1 La ciencia para el falsacionismo La necesidad de determinar una línea objetiva para separar el conocimiento científico del no científico, llevó a Popper a proponer su criterio de demarcación y formular su teoría del falsacionismo, dando origen a una de las escuelas más prometedoras del siglo XX. La tesis Popperiana sostiene que no se empieza por observaciones sino, siempre a partir de problemas o a partir de una teoría que ha pasado por serias dificultades, es decir, una teoría que ha creado y decepcionado determinadas expectativas. La ciencia es considerada como un conjunto de hipótesis -que deberán ser probadas- para explicar o describir aspectos del mundo. Si bien, no todas las hipótesis pueden hacerlo, todas deben ser falsables. Para el falsacionismo, el progreso de la ciencia se da ante el planteamiento de problemas.

Popper (1935) analiza, todavía desde un marco de referencia predominantemente lógico, las reglas del juego en la obtención de hipótesis y elaboración de teorías científicas. La investigación científica tendría como meta y problema central el crecimiento del saber científico. Popper, considera que la tesis central del positivismo lógico como una perspectiva reduccionista y no cree que para estudiar tal desarrollo del saber sea suficiente (y posible) reducirse al estudio de los lenguajes artificiales, o al de los cálculos lógicos desarrollados en ellos.

La concepción falsacionista sofisticada de la ciencia plantea una imagen dinámica de la misma, al no preguntarse ya sobre la posibilidad de una teoría de ser falsable o la medida en que lo es, sino al proponer si la teoría recién propuesta puede sustituir a la predecesora. De este modo toda teoría que sea más falsable y que prediga fenómenos que su rival no consideraba, es digna de atención.

\subsection{La ciencia como estructura organizada} Thomas Kuhn introduce la noción de paradigma, el cual está constituido por supuestos teóricos, leyes y técnicas de aplicación que deberán adoptar los científicos que se mueven dentro de una determinada comunidad científica. Para Kuhn, la existencia de un paradigma que pueda apoyar una tradición de ciencia normal será lo que establecerá la diferencia entre lo que es ciencia y lo que no lo es. Carecer de paradigma implica no poseer el estatus de ciencia. La ciencia normal es descrita por Kuhn como una actividad de resolver problemas gobernada por las reglas de un paradigma en cuestión. El paradigma deberá proveer los medios para solucionar los problemas que en él se formulan.

Los que trabajan dentro de un paradigma ponen en práctica la ciencia normal. Es probable que al trabajar en ella, el paradigma se desarrolle en su intento por explicar los comportamientos de los aspectos del mundo y resulten algunas dificultades. Si estas se vuelven inmanejables, se desarrollara un estado de crisis, la cual se resolverá con el surgimiento de un nuevo paradigma, el cual cobrará cada vez mayor adhesión por parte de la comunidad científica. 
Imre Lakatos desarrollo su concepción de la ciencia en programas de investigación, considerando a las teorías como estructuras organizadas. Esta estructura tiene su utilidad ya que se constituiría en una guía, tanto positiva como negativamente para la futura investigación. Estos programas serán progresistas si conducen a descubrir nuevos fenómenos y serán degeneradores si no lo hacen.

\section{Definición de ciencia}

De acuerdo con lo señalado por Tamayo (1999), ciencia es aquel "conjunto de conocimientos racionales, ciertos y probables, obtenidos metódicamente, sistematizados $\mathrm{y}$ verificables, que hacen referencia a objetos de una misma naturaleza". A partir de esta definición debemos entender que estos conocimientos están referidos a la realidad (mundo), y a los hechos y fenómenos que en ella acontecen. Constituye un quehacer crítico no dogmático, debido a que somete a todos sus supuestos a ensayo y crítica, buscando siempre establecer relaciones existentes entre los diversos hechos, e interconectarlos entre sí, a fin de lograr conexiones lógicas que permitan presentar postulados o axiomas en los distintos niveles del conocimiento; los cuales sistematiza utilizando la investigación, determinando la objetividad de las relaciones que establece entre los hechos y fenómenos de que se ocupa a través del método científico.

\section{Elementos o características de la cien- cia}

Establecer los aspectos característicos de la ciencia obliga a establecer una comparación con la ciencia antigua, medieval y la moderna. Por un lado la ciencia antigua parte de los hechos y la segunda parte de los conceptos generales y especulativos, sin embargo, lo decisivo y característico de la ciencia moderna es el modo en que los hechos son comprendidos y los conceptos aplicados. En este sentido, no es el experimento como tal lo característico de la ciencia moderna sino el modo en que se proyecta el experimento para determinar conceptualmente los hechos. Sin embargo, no debemos olvidar que el problema también radica en el modo y el sentido de los cálculos y mediciones.

Estas son las características de la ciencia moderna, ciencia de los hechos, ciencia experimental, y de la medición; sobre las que Heidegger (1960) observa señalando que la nueva exigencia del saber es "la exigencia matemática”.

\subsection{El Objeto de estudio de la ciencia}

En las teorías del conocimiento clásicas es usual la distinción entre "sujeto" y "objeto". El sujeto se refiere al agente del conocimiento, 'al que conoce', y el objeto a 'lo que se estudia y conoce.' Decimos que el 'conocimiento' es lo que media entre el uno y el otro. El conocimiento de un objeto, que puede ser un fenómeno, hecho social, realidad, etc., empieza por la comprensión del mismo, por esta razón, los estudiosos han partido de diversas formas de aproximación, las cuales evidencian lo complejo que es la realidad y las diferentes formas de entenderla y aprehenderla. La ciencia, brinda un acercamiento tanto a la realidad asentida como a la realidad experimentada (Babbie, 2000) y otorga una respuesta a la pregunta ¿Cómo saber lo que es real en realidad? La idea de lo que es la realidad ha sido concebida de manera distinta a lo largo de la historia. Así pues, la idea pre-moderna de la realidad y que ha guiado casi toda la historia, presuponía que los objetos se veían como eran realmente. A medida que evolucionamos y nos percatamos de la diversidad, llegamos a aceptar que los demás no siempre comparten nuestras opiniones. 
Es entonces cuando se legitima la diversidad constituyéndose como la idea moderna de la realidad. Aquí se acepta el carácter inevitable de la subjetividad humana. La idea postmoderna de realidad se basa en lo siguiente: todo lo "real" son imágenes que recibimos por nuestros puntos de vista. Para decirlo de otra manera, no hay nada fuera, todo está dentro. La idea postmoderna representa un dilema crítico para los científicos. Su tarea es observar y entender lo que "realmente" pasa, pero son seres humanos, y como tales, poseen inclinaciones personales que matizaran inevitablemente lo que observan y cómo lo explican (Babbie, 2000). El conocer es enfrentar la realidad, es una actividad por medio de la cual el hombre adquiere certeza de la realidad, y que se manifiesta como un conjunto de representaciones sobre la cuales tenemos certeza de que son verdaderos (Tamayo, 1999).

Por ello, la ciencia moderna espera la mayor objetividad posible del sujeto frente al objeto de conocimiento, es decir, se debe tratar de eliminar la mayor subjetividad posible y de esta manera garantizar el conocimiento científico. Sin embargo, tal pretensión de objetividad responde más a un ideal que a una realidad pues son muchos los aspectos que la dificultan. Un objeto de investigación es definido y construido en función de una problemática teórica, que implica a su vez aproximaciones metodológicas constantes, y tratar a los hechos no de manera aislada, sino en función de relaciones establecidas entre ellos; por ejemplo, hacer surgir propiedades ocultas que no se revelan sino en el enlace de cada una de las realizaciones con todas las otras. A su vez, la construcción de un objeto de investigación exige una actitud crítica, fundamentada en la historia (Guzmán, 1992). Entonces, se puede concluir que el objeto de conocimiento ni se deduce directamente de "esencias" del mundo de las ideas, ni se aprehende directamente de la percepción, sino que se construye por medio de múltiples operaciones lógicas, teóricas, metodológico-técnicas, praxeológicas... (Sánchez, 2007)2.

Todo conocimiento sobre un objeto, es forzosamente una relación en la cual aparecen dos elementos relacionados entre sí; uno cognoscente, llamado sujeto, y otro conocido, llamado objeto. Esta relación implica una actividad en el sujeto, la cual es la de aprehender el objeto, y la del objeto es simplemente de ser aprehendido por el sujeto. Es pues, el sujeto quien determina la relación con el objeto, y por tanto determina esa actividad de conocer y puede entrar en relación con el objeto de diferentes maneras, lo cual hace que la actividad de conocer fluctúe entre el conocimiento vulgar y el conocimiento científico. Existen características diferenciadoras entre estas ciencias, por ejemplo, las ciencias sociales con respecto a las ciencias naturales, se diferencian en que los seres humanos poseen habilidades cognitivas específicas que crean una conciencia y representaciones mentales abstractas que en general influyen en su comportamiento y crean unas reglas de interacción entre individuos complejas, por tanto, introducen hechos mentales reales o supuestos. Por otro lado las ciencias sociales se diferencian de las humanidades, en que estas dan un mayor énfasis al método científico u otras metodologías rigurosas de análisis. De esta última, diferenciación, se puede advertir que existen también similitudes en las distintas ciencias mencionadas. Por ejemplo,

2 Entrevista contenida en: El Objeto de conocimiento en la ciencia. Diversas aproximaciones. 
que las ciencias sociales usa el método científico en forma rigurosa, conforme lo utilizan las ciencias naturales.

\subsection{El Método de la ciencia}

A lo largo de la historia de la ciencia e incluso hasta nuestros días, se ha evidenciado siempre la presencia o la relación, entre el método como camino y la experiencia de búsqueda del conocimiento entendida como travesía generadora de conocimiento y sabiduría. ....Por esta razón el método no precede a la experiencia, el método emerge durante la experiencia y se presenta al final, tal vez para un nuevo viaje ${ }^{3}$.

Aunque existen criterios opuestos con relación al método, como el manifestado por Feyarebend (2009), quien sostiene que: No existe un método único para la ciencia. ... no hay ningún "método científico", no hay un procedimiento único o un conjunto de reglas que presidan todo trabajo de investigación y garanticen que un trabajo es "científico" y, por tanto, digno de confianza ${ }^{4}$.... Tampoco (el método) está oculto en el proceso progresivo de la ciencia, ni requiere para su asimilación hundirse en este proceso o participar en él...es más un mito y no una determinación fundamentada de la naturaleza de la ciencia, que subyace en la exigencia de los expertos con el propósito de hacerse de poderes especiales, oponiéndose y eliminado de esta manera toda idea extra-científica ${ }^{5}$.... No hay una sola regla, por plausible que sea, ni por firmemente basada en la epistemología que venga, que no sea infringida en una ocasión o en otra ${ }^{6}$... Los métodos solo funcionan en determinados ámbitos, mientras que en otros fracasan ${ }^{7}$. La idea de un método fijo, de una (teoría de la) racionalidad fija, surge de una visión del hombre y de su contorno social demasiado ingenua .... hay solamente un principio que puede ser defendido bajo cualquier circunstancia y en todas las etapas del desarrollo humano. Este principio es "todo vale" ${ }^{8}$. Este principio es el único de la metodología anarquista de Feyerabend.

Morin, menciona también que existe una relación entre la teoría y el método, precisando que "...una teoría no es la solución, es la posibilidad de tratar un problema. Una teoría sólo cumple su papel cognitivo, solo adquiere vida, con el pleno empleo de la actividad mental del sujeto. $Y$ es esta intervención del sujeto lo que le confiere al método su papel indispensable. Identifica una relación recursiva entre método y teoría. El método, generado por la teoría, la regenera" ${ }^{\text {. Señala }}$ además que método y paradigma son inseparables. Toda actividad metódica está en función de un paradigma que dirige una praxis cognitiva ${ }^{10}$.

Zambrano (1989), al precisar la relación entre la experiencia y el método señala que: la experiencia precede a todo método, es decir la experiencia es a priori y el método es a pos-

3 MORIN y otros (2003), Educar en la era planetaria, Capitulo I: El Método, Editorial Gedisa, p.20.

FEYERABEND, Paul (2009). ¿Por Que no Platón?, Edit. Tecnos, 3ra edición, Madrid, España, p. 93.

5 Idem 25, p. 52.

6 FEYERABEND, Paul (2009). Contra el Método, p. 18-52. En: MAZZI HUAYCUCHO, Victor (2006); Epistemologia- selección de lecturas, Edit. San Marcos, p. 688-689.

7 Idem 14, p. 73.

8 Idem 16, p. 692.

9 Idem 19. p. 25

10 Idem 19, p. 43.

11 Zambrano, M. Notas de un Método, Mandadori, Madrid 1989.

12 Idem 19. 
teriori ${ }^{11}$. $\mathrm{Al}$ respecto, Morin precisa que esto es solo una indicación, ya que la verdadera experiencia no puede darse sin la intervención de una especie de método ${ }^{12}$.

\subsection{La capacidad de predicción de la cien- cia}

Cualesquiera que sean los impulsos o instintos primitivos que motiven a los seres humanos y otros animales, su satisfacción depende en gran medida de la capacidad de predecir circunstancias (Babbie, 2000), pero debemos situar nuestros esfuerzos de predicción en el contexto del conocimiento y la comprensión de ciertos fenómenos.

Por lo general la predicción se refiere a un enunciado, una oración descriptiva acerca de un suceso, mayormente de bajo nivel de generalidad. En las ciencias empíricas, una predicción se puede entender de dos maneras: como la operación o actividad de formular una descripción de un hecho desconocido o como la descripción misma, o sea el enunciado. La capacidad de predecir con certeza y precisión el comportamiento futuro de un sistema es una de las máximas aspiraciones de la ciencia clásica, con sólo conocer la definición de uno de los estados del objeto considerado y la ley que rige su evolución. En el mundo real y práctico, El propósito primario de la propia ciencia es alcanzar una capacidad de predicción lo cual nos dará algún control sobre nuestras vidas y una cierta protección del ambiente que nos rodea, evitando de este modo los daños del mundo, conociendo donde y cuando van a ocurrir ${ }^{13}$. Por tanto, predecir es la verdadera esencia de la ciencia, la cual determina si una teoría es científica. Toda predicción científica, al ser formulada como un enunciado, hace permanecer como una conjetura su estatus cognitivo, o sea que es una hipótesis cuyo valor de verdad no conocemos todavía. En consecuencia, es un error identificar la predicción con la descripción de un suceso futuro, pues no toda predicción se refiere al futuro. Por tanto, predicción científica, es sinónimo de hipótesis, conjetura.

Una predicción científica se formula a partir del conocimiento teórico -generalmente basado en leyes- que se posee en una cierta disciplina científica, por ello no es caprichosa ni una mera profecía. Pero lo que diferencia una predicción científica de una profecía es que una predicción es un enunciado desconocido (en cuanto a su valor de verdad) que se formula a partir de otros conocidos, de manera condicional y justificada. Nótese que esta definición no hace referencia al momento de la ocurrencia del hecho, pues se trata de un concepto epistemológico, cognitivo, y no metafísico. Por ejemplo, si se parte de que todos los metales se dilatan cuando son calentados -una ley- y se toma una vara de metal para luego calentarla -las condiciones iniciales- se puede luego predecir "esta vara de metal se dilató". Nótese que el hecho ya ha ocurrido, es pasado que se dilató, pero se trata de una auténtica predicción, un pasaje de lo conocido a lo desconocido, que luego deberá ser contrastado con la observación o el experimento (mediremos la vara para comparar el resultado de la medición con un valor anterior). Similarmente, si decido alterar mi dieta regular con abundantes comidas calóricas, luego de un par de días, si no me he pesado, podré formular la predicción de que engordé. De manera que lo que siempre ocurre después de una predicción es la prueba o test de la predicción, y no el hecho. 


\subsubsection{Las predicciones en las ciencias so- ciales}

Tomando el modelo a las ciencias naturales, diremos que la producción de las ciencias sociales consiste en proveer el conocimiento de regularidades en los comportamientos, que expliciten las expectativas grupales o sociales, permitiendo realizar predicciones, con la más alta probabilidad posible, sobre tendencias y patrones de comportamiento en cada ámbito de estudio. Podrían a su vez elaborarse procedimientos para generar o transformar conductas conforme a determinados intereses y fines. Tales elaboraciones permitirían diseñar una ingeniería social (un conjunto articulado de tecnologías sociales), con la que se intentaría controlar con eficacia las acciones de los actores sociales. De esta manera, como las ciencias naturales, las ciencias sociales explorarían soluciones de problemas prácticos, proveyendo patrones $\mathrm{y}$ tendencias de comportamiento; haciendo posible desarrollar planificadamente una intervención social que permite el control de las interacciones.

Las investigaciones cuantitativas utilizan las encuestas y las estadísticas tratando de captar las recurrencias, es decir, los comportamientos que se repiten con mayor proporción en una población. Las cualitativas exploran los modos en que los actores sociales significan sus prácticas. Reconstruyen los significados que guían las conductas del grupo. En ambos casos, los resultados de esas investigaciones permiten realizar predicciones de las conductas de esos actores, basadas en esas recurrencias y en esos significados.

Estas predicciones no son como leyes mecánicas de la física, no suponen uniformidad ni provocan reiteración idéntica. Solo afirman patrones de conducta y tendencias, debido a que los actores poseen algún grado de libertad. Ello no hace posible predeterminar totalmente las conductas humanas por los patrones y las tendencias del comportamiento. Por otro lado, los frutos más sobresalientes que se le atribuyen a las ciencias naturales (duras), es la capacidad de predicción. Así y todo, hoy en día, predecir un gran terremoto sigue siendo muy complicado: la escala y el número de variables que están en juego son enormes. Pero, con el tiempo -ese gran constructor- la información será más abundante y precisa y las predicciones serán mejores y, entre otras cosas, ayudarán a salvar vidas.

Resulta entonces que ajustándonos al criterio de cientificidad dominante se manifiesta la debilidad de la capacidad predictiva de las ciencias sociales. Es una debilidad relativa, ya que surge de la comparación con las fortalezas de la capacidad de predicción de las ciencias naturales. Finalmente, debemos reconocer señalar que la capacidad de predicción de las Ciencias naturales, difiere de Ciencias sociales en la clase de predicción que cada una produce. Por tanto, quienes acusan la cientificidad de las ciencias sociales deberían precisar las razones de sus críticas justificando el reclamo de una mayor y mejor capacidad predictiva, dando cuenta de la especificidad de la producción del conocimiento sobre la sociedad.

\section{DISCUSIÓN DE RESULTADOS}

La ciencia es un término acuñado con el devenir del tiempo, aunque inicialmente lo constituían aquellos conocimientos fijos e inmutables, conforme lo señala Platón, los cuales eran el resultado de un proceso de abstracción, sustentada en una necesidad ontológica ficticia y apriorística. Este proceso racional, estaba influido por la subjetividad del individuo. Por esta razón, Aristóteles conserva el concepto platónico de la ciencia como conocimiento, fijo, estable y necesario, 
pero la necesidad la busca en el orden lógico. La tesis aristotélica consideraba ciencia a todo producto elaborado por el entendimiento, y con valor lógico; a través del proceso de abstracción, lo que constituía su método. La definición aristotélica de ciencia, basada en la observación, suprime algunas ideas platónicas trascendentes, admitiendo solo las sustancias particulares divididas jerárquicamente en tres planos: terrestre, celeste y divino. Suprime también las nociones de participación e imitación. Sin embargo, logra distinguir dos órdenes de conocimiento: el sensitivo y el intelectivo (Fraile, s/f). Aunque los silogismos son poderosos instrumentos para examinar el razonamiento científico, no nos dicen nada o no están diseñados para hacerlo, sobre el contenido de verdad de las premisas, sino que constituyen simples reglas de Lógica para usarse una vez que las premisas se han alcanzado (Pérez, 2010).

Para los empiristas, las ciencias naturales son el tipo ideal de ciencia, ya que se basa en hechos observables. Sostienen que sin el contacto con la naturaleza, y para que el método científico no se detenga y se continué con la deducción, es necesario determinar cuál es la realidad verdadera. Aunque esto implicaría de algún modo se estaría eliminando el riesgo de construir premisas sin contenido de verdad, con el costo de reducir el contenido de la ciencia a aquellas cualidades que pueden expresarse matemáticamente y compararse en forma de relaciones. También sostienen que la primera manera de saber es a través de la experiencia, pero el deseo de saber no se detiene allí, ya que el hombre pretende saber y conocer la esencia de las cosas, de por qué se desenvuelven de una manera y no de otra.

Ciencia para los empiristas es pues, un conjunto de conocimientos obtenidos de una serie de hechos individuales, que han sido confirmados a través de la experimentación, sobre los cuales recae un proceso de abstracción riguroso que permite obtener conceptos generales de las cosas y las leyes de la naturaleza. Los métodos utilizados eran el experimental y el inductivo. Adviértase aquí, que la utilización de la experiencia como medio para conocer, exige la capacidad de predicción del conocimiento científico.

La ciencia aquí es deducida de los principios del entendimiento y la extensión, las causas de todo lo que acontece, emulando a las matemáticas que permiten deducir sus premisas. El medio para hacerlo era el pensamiento, como criterio de certeza.

Una crítica que podemos imputarle al empirismo es que restringe la capacidad de la mente humana, limitándola a la experiencia, es decir, más allá de ella no es lícito ir si no queremos caer en el error, atribuyéndole a todo lo que no ha sido "experimentado" una realidad y existencia objetiva.

La concepción de ciencia para los racionalistas, es aquella que se inicia con la observación y la componen un conjunto de enunciados básicos o protocolares, cuya certeza viene dada por la percepción inmediata de los sentidos, es decir la ciencia se define por los términos sacados de la experiencia sensible. El conocimiento procede de la razón lo que va asociado a la afirmación de la existencia de ideas innatas. Ello constituye el punto de partida de la teoría gnoseológica o epistemológica. Esta perspectiva hace prevalecer como explicación científica la explicación causal, buscando la esencia matemática que permita la predicción de los fenómenos. Sostienen la necesidad de usar el método de las ciencias naturales (deductivo), para eliminar la variedad de opiniones y teorías contrarias. 
El positivismo concibe a la ciencia como un conjunto de enunciados básicos o protocolares, cuya certeza viene dada por la percepción inmediata de los sentidos. La ciencia se define por los términos sacados de la experiencia sensible. Esta corriente busca la esencia matemática que permita la predicción de los fenómenos. Ellos utilizan el método inductivo al que denominan el método de las ciencias, el cual debería servir a las demás ciencias. A esto se le denomina como el monismo metodológico.

Desde la perspectiva del racionalismo crítico, Popper como uno de los exponentes de esta corriente, señala que la formación del conocimiento es un proceso evolutivo que parte de problemas y tiene intentos de solución y exclusión de intentos fallidos; haciendo uso del método deductivo. La ciencia es considerada como un conjunto de hipótesis -que deberán ser probadas- para explicar o describir aspectos del mundo. Si bien, no todas las hipótesis pueden hacerlo, todas deben ser falsables. Para el falsacionismo, el progreso de la ciencia se da ante el planteamiento de problemas. Kuhn, otro representante del racionalismo crítico, afirma que la existencia de un paradigma que pueda apoyar una tradición de ciencia normal será lo que establecerá la diferencia entre lo que es ciencia y lo que no lo es. Carecer de paradigma implica no poseer el estatus de ciencia. La ciencia normal es descrita por Kuhn como una actividad de resolver problemas gobernada por las reglas de un paradigma en cuestión. El paradigma deberá proveer los medios para solucionar los problemas que en él se formulan.

\section{Conclusiones}

1. La concepción de ciencia ha estado influenciada por las diversas corrientes de pensamiento, las cuales al privilegiar cierto método, mostraban vaivenes en cuanto al método de la ciencias, cuyas consecuencias han sido mayor o menor objetividad en la obtención del conocimiento y dando lugar a la formulación de opiniones como la de Feyarebend, respecto a que no existe un método de la ciencia.

2. Independiente de cual fuere la corriente de pensamiento, todas ellas han mostrado a lo largo de su proceso evolutivo que la definición de ciencia contiene algunos elementos o componentes comunes que caracterizan a toda disciplina científica. Estos elementos comunes que otorgan el estatus de ciencia a una disciplina son: el objeto de estudio, el método y la capacidad de predicción. Aunque, Kuhn agrega un requerimiento adicional para que cualquier disciplina pueda alcanzar el estatus de ciencia: la existencia de un paradigma.

\section{REFERENCIAS BIBLIOGRÁFICAS}

1.- ALONSO, Carlos Javier (2001). Historia Básica de la Ciencia- pasión por el saber-. España: Edit. EUNSA (Ediciones Universidad de Navarra S.A.).

2.- BELLO REGUERA, Eduardo (2006). El Discurso del Método: Estudio preliminar. Madrid, España: Edit. Tecnos, Sexta edición.

3.- BUNGE, Mario (1960). La ciencia: su método y su filosofía. Ariel, Buenos Aires, Argentina.

4.- SABOGAL AQUINO, Mario (2005). Comunicación y Epistemología, Módulo I Una comprensión epistemológica de la comunicación, Compendio ordenado. Facultad de Ciencia Histórico Sociales y 
Educación, Universidad Nacional "Pedro Ruíz Gallo”, Lambayeque, Perú.

5.- CAVADA, Daniel (2004). Ciencias Naturales y Ciencias Sociales: Un debate sobre su acercamiento inter-paradigmático, Tesis para optar el grado de sociólogo, Chile.

6.- DOMINGUEZ G., Silvia (2007). “Objeto de estudio en la investigación: diversas aproximaciones", Revista de Educación y Desarrollo, $\mathrm{N}^{\circ} 7$.

7.- ECHEVARRIA, J. (2002). Ciencia y Valores. Barcelona: Destinos.
8.- FRAILE, Guillermo, Historia de la filosofía. España: Tomo I, Colección de la BAC.

9.- GOMEZ VILLEGAS, Mauricio (2003). Comentarios sobre el discurso científico y los determinantes morales. Colombia: FENECOP.

10.- CHALMERS, ALAN F. (1992). La ciencia y cómo se elabora, Madrid, Siglo XXI. 